\title{
The Relationship Between Implicit Preference Between High-Calorie Foods and Dietary Lapse Types in a Behavioral Weight Loss Program
}

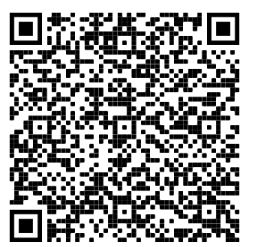

\author{
Clare Jocelyn Mangubat ${ }^{1 *}$, Rebecca J. Crochiere ${ }^{2}$, Evan M. Forman ${ }^{3}$
}

Weight loss failure is driven by dietary lapses, i.e., instances of non-adherence to a dietary prescription. Lapses can occur when an individual eats a larger portion than intended, a type of food intended to be avoided, or food at an unintended time. It is important to identify determinants of dietary lapses to tailor weight loss treatment. Higher implicit preference for high-calorie, hedonic food may be a predictor of each of these lapse types. The aim of the study was to examine if baseline implicit preference for high-calorie food is positively associated with large portion, unintended food, and unintended time dietary lapses. Participants $(N=189)$ from a behavioral weight loss trial followed a weight-loss diet and reported six times/day if a lapse occurred via smartphone-delivered surveys. Baseline implicit preference for high-calorie food was measured using the Implicit Association Test (IAT). There was a statistically significant positive correlation between IAT and unintended time lapses $(r=0.15, p=0.047)$, such that increases in IAT were weakly associated with increases in proportion of unintended time lapses. The study provides preliminary evidence that individuals with higher implicit preference may be susceptible to eating at unintended times, perhaps due to strong approach tendencies when food stimuli are present. These findings can inform individualized tailoring of weight loss interventions by teaching at-risk individuals strategies to prevent eating at unintended times.

\section{INTRODUCTION}

In the United States, $70 \%$ of adults have overweight or obesity (Centers for Disease Control and Prevention, 2017). Obesity and overweight are linked to various health problems, including cardiovascular disease, increased risk for mental illness, and decreased quality of life (Pulgaron, E. R., 2013; Williams et al., 2015; Caleyachetty et al., 2017). Many individuals with overweight or obesity follow a dietary prescription to lose weight, but typically, individuals do not follow these diets consistently. These momentary breaks in diets, called dietary lapses, contribute to weight-loss failure (Lowe, 2003; Forman et al., 2017). Dietary lapses may occur because an individual eats a larger portion of a meal or snack than intended (larger portion), eats a specific food

Address correspondance to:

'School of Nursing, University of Pennsylvania, 418 Curie Boulevard, Philadelphia, PA 19104, United States

${ }^{2}$ Center for Weight, Eating, and Lifestyle Sciences

(WELL Center), Drexel University, 3201 Chestnut Street

Philadelphia, PA 19104, United States

${ }^{3}$ Department of Psychology, Drexel

University, 3201 Chestnut Street Phila-

delphia, PA 19104, United States

*cjpm2828@gmail.com

Submission date: August 2020

Acceptance date: December 2020

Publication date: March 2021 doi:10.22186/jyi.39.3.27-31

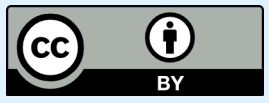

Except where otherwise noted, this work is licensed mons.org/licenses/by/4.0 under $h$ ttps://creativecom item intended to be avoided (unintended food), or eats food at an unintended time outside of designated meal and snack times (unintended time). To improve weight-loss outcomes with dietary prescriptions, dietary lapses must be minimized. This can be accomplished by better understanding dietary lapses so that more effective interventions targeting dietary lapses can be developed (Forman et al., 2018).

Research has found that momentary factors may predict dietary lapse by using ecological momentary assessment (EMA). EMA is a data collection method that gathers user feedback in an individual's natural environment, usually via a smartphone. Various physical (e.g., deprivation) and affective states (e.g., loneliness) have been identified as triggers of lapses (Carels et al., 2001; Carels et al., 2004; Forman et al., 2017). Furthermore, elevations in feelings of deprivation, hunger, and boredom have been found to increase the risk of different dietary lapse types (e.g., momentary increases in deprivation and hunger increased the likelihood of experiencing an unintended food dietary lapse) (Goldstein et al., 2018).

Although relations between self-reported factors (e.g., affective state, stress, hunger) and subsequent dietary lapses have been explored, less research has examined how automatic processes, including implicit preference, influence risk of lapsing from a dietary prescription. Several studies show that implicit preferences are linked to different types of dysregulated eating. For example, Nederkoorn et al. (2010) found that among individuals with high implicit preferences for high calorie snack foods, participants with lower inhibi- 
tion gained more weight after one year than participants with higher inhibition. Among participants with low implicit preference for snack foods, there was no association between inhibition and weight gain (Nederkoorn et al., 2010). Research has also shown that higher implicit preference for high-fat, sweet foods is associated with overcompensating after exercise (i.e., eating more food following exercise compared to the amount eaten without exercising) (Finlayson et al., 2009). Furthermore, previous studies have found that automatic processes drive spontaneous decisions when offering participants the choice between a hedonic snack (e.g., pastries, chocolate cake) and fruit (Shiv and Fedhorikin 1999; Shiv and Fedhorikin, 2002; Perugini, 2005). When participants were cognitively preoccupied and made the food decision spontaneously, they chose the hedonic snack (Shiv and Fedhorikin 1999; Shiv and Fedhorikin, 2002). These studies suggest that implicit preferences for high calorie food might be linked to weight gain, compensatory eating, and spontaneous food choices. Given these findings, implicit preferences may also be related to dysregulated eating that lead to dietary lapses. However, no studies have examined the role of automatic processes on dietary lapse likelihood within the context of a behavioral weight loss intervention.

In the obesogenic environment, where highly palatable food (e.g., chocolate cake) is ubiquitous and easily accessible, implicit preference for high-calorie food may make it more difficult for people to adhere to their dietary prescriptions. A person with a strong implicit preference for palatable food may have more difficulty eating only an intended portion while eating a meal or while snacking because of how enjoyable they perceive the food to be. As such, the individual may have a strong desire to add more food to his plate, thus raising their risk of experiencing a larger portion dietary lapse. Likewise, an individual with a strong implicit preference for palatable food may have more difficulty only eating three meals and two snacks, as prescribed, than an individual with a weak implicit preference for palatable food. They may be continually tempted by food throughout the day in the obesogenic environment. As such, they may experience a strong desire to eat food they are exposed to outside of meal and snack times, increasing the risk of experiencing an unintended time dietary lapse. Additionally, with many opportunities to eat food intended to be avoided in the obesogenic environment (e.g., lunch-ins at work, fast-food restaurants on the drive home), a person with higher implicit preferences for high-calorie food may have a strong desire to choose hedonic food instead of healthy food, raising the risk of experiencing an unintended food dietary lapse.

The aim of the study was to examine if baseline implicit preference for high-calorie food was positively associated with large portion, unintended food, and unintended time dietary lapses. This study is the first to explore the association between baseline implicit preference for palatable foods and dietary lapse types (i.e., larger portion lapses, unintended food lapses, and unintended time lapses) among partici- pants with overweight/obesity following a behavioral weight loss program.

\section{METHODS}

\section{Participants}

This is a secondary analysis examining data from baseline and the first two weeks of a larger behavioral weight loss trial (Forman et al., 2016). $189(N=189)$ adults with overweight or obesity $\left(82.0 \%\right.$ female; $70.9 \%$ Caucasian; $\mathrm{M}_{\text {age }}=51.8 \pm$ 9.8 years; $M_{B M I}=36.9 \pm 5.8 \mathrm{~kg} / \mathrm{m}^{2}$ ) were recruited from the Philadelphia area. At baseline, informed consent from participants was obtained.

\section{Procedures}

All participants were randomized to one of two group behavioral weight loss interventions for 12 months. At baseline, the Implicit Association Test was administered. Participants were prescribed a daily calorie goal and were informed that exceeding this goal would presumably impede weight loss and be a dietary lapse. For the first two weeks of the study, participants followed an EMA protocol by answering surveys about dietary lapse types six times a day on Android devices. Because the interventions were identical during the first two weeks of treatment for both conditions, the EMA data were collapsed across conditions. Study procedures were reviewed and approved by Drexel University's Institutional Review Board.

\section{Measures}

\section{Lapse Type}

Participants were given Android smartphones (Samsung Galaxy Player 4.0) with the application DrexelEMA. This application sent semi-random surveys six times a day asking whether a dietary lapse occurred and, if it did, the type of lapse. The options for dietary lapse type included larger portion (i.e., eating a larger portion than intended), unintended food (i.e., eating specific food that was intended to be avoided), and unintended time (i.e., eating food at an unintended time). Participants were taught how to use DrexelEMA and how to recognize dietary lapses. They were instructed to complete the six EMA surveys every day and to manually report lapses immediately after they occurred.

\section{Implicit Preference for High-Calorie Food}

Implicit preference for hedonic food was measured with the IAT at baseline. In this task, automatic associations between categories and characteristics were assessed by having participants instantly respond to images on a computer. Participants were instructed to sort two food categories (lowcalorie and high-calorie) into two affective categories ("good" versus "bad") using corresponding keys ("e" or "i") as quickly and accurately as they could.

Participants sorted images from one food category (e.g., low-calorie) and images of positive stimuli into the "good" 
category using one response key while they sorted images from the other food category (e.g., high-calorie) and images of negative stimuli into the "bad" category using the other response key. Then, participants sorted the opposite combination of categories and characteristics (e.g., low-calorie with "bad" and high-calorie with "good"). The higher the IAT score, the higher the implicit preference for high-calorie food (i.e., the greater the association of highly palatable food as "good" compared to highly-calorie food as "bad"). Previous studies have found that the IAT has good construct validity (Nosek et al., 2005) along with internal consistency (0.80) Banse et al., 2001; Egloff and Schmukle, 2002).

\section{Analyses}

Analyses were conducted in SPSS v. 25 and in R v. 3.5.3. Pearson's correlation tests were used to assess the relation between baseline IAT scores and proportion of each lapse type, which is the number of each lapse type out of all participant surveys. The proportion of lapse type was used rather than the raw number of lapse type to control for each participant's adherence to the EMA protocol. In doing so, the study controlled for the effect that higher compliance (i.e., people reporting more lapses because they are more compliant with the EMA protocol) may have on the relationship between baseline IAT scores and dietary lapses and on the higher likelihood for reporting a lapse overall. Hotelling-Williams Test was conducted to compare Pearson's correlations between IAT scores and each lapse type.

\section{RESULTS}

\section{EMA Adherence and Data Descriptives}

The mean EMA survey adherence was $82.4 \%(S D=13.3 \%)$. Individuals were excluded from analyses if their survey adherence was below $40 \%(n=3)$. The sample used in statistical analyses was $N=186$. From these 186 participants, 13,280 EMA surveys were collected.

\section{Associations Between Implicit Preference for High-Calorie Food and Dietary Lapse Types}

Contrary to expectation, no association was observed between either IAT and unintended food lapses $(r=-0.03, p=$ $0.734)$ or between IAT and larger portions lapses $(r=-0.03$, $p=0.678)$. The study found a statistically significant positive correlation between IAT and unintended time lapses $(r$ $=0.15, p=0.047$ ), such that increases in IAT were weakly associated with increases in proportion of unintended time lapses out of all participant surveys (Figure 1). The highest bin of baseline IAT scores appears to have the highest proportion of unintended time lapses out of all EMA reports. Hotelling-Williams Test indicated that the correlation between IAT and unintended time lapses was not significantly higher than the correlation between IAT and Unintended Food or larger portion lapses $(t(189)=1.73 ; p<.09)$.

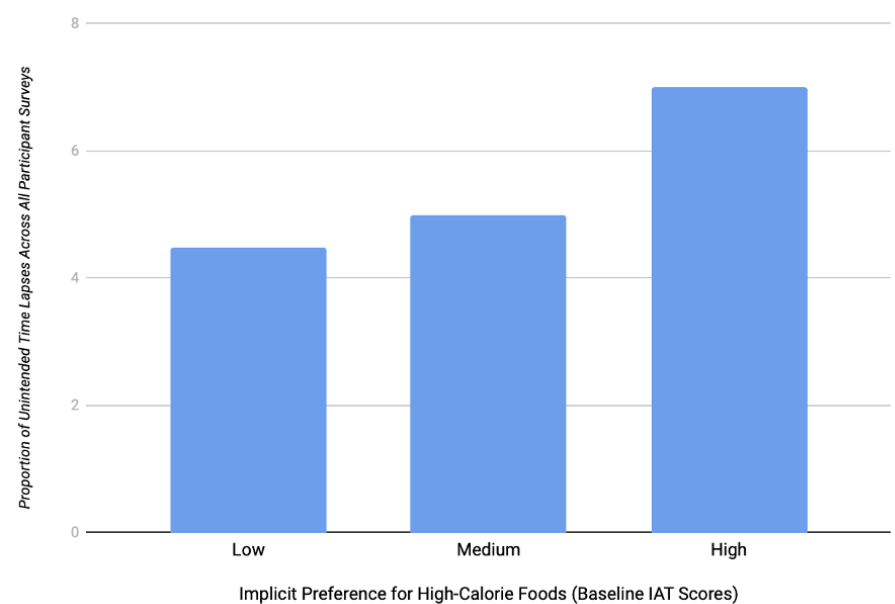

Figure 1. Proportion of Unintended Time Lapses by Implicit Preference for High-Calorie Foods (Baseline IAT Scores). The proportion of unintended time lapses out of all participant surveys was calculated to control for the effect that higher participant adherence to the EMA protocol (i.e., people reporting more lapses because they are more compliant with the EMA protocol) may have on the relationship between baseline IAT scores and dietary lapses and on the higher likelihood for reporting a lapse overall.

\section{DISCUSSION}

The current study investigated and compared the relationships between implicit preference for high-calorie food and dietary lapse types. It was hypothesized that baseline implicit preference for hedonic food would be associated with unintended food, larger portion, and unintended time lapses. Surprisingly, implicit preference was not statistically significantly correlated with eating larger portions than intended nor with eating food intended to be avoided within the behavioral weight loss program. Implicit preference for hedonic food was weakly associated with eating at an unintended time while dieting, which weakly supports our hypothesis. However, this correlation was not significantly larger than the other correlations.

The current study provides the first evidence that higher implicit preference for high-calorie food may be associated with unintended time dietary lapses. In other words, individuals with an unconscious liking for hedonic foods may be more at risk for breaking a dietary prescription by eating at an unintended time. Understanding how individual differences can put participants at increased risk of different dietary lapses can inform tailoring of personalized treatment such that atrisk individuals can be taught specific coping skills to avoid lapses. It may be that individuals with strong implicit preferences for high-calorie foods are particularly susceptible to eating at unintended times, perhaps due to strong approach tendencies (i.e., the tendency to automatically approach a stimulus rather than avoid it) when in the presence of food stimuli. Kemps and Tiggemann (2015) found that individuals with obesity have a stronger approach tendency toward both 
high-calorie and low-calorie food than individuals without obesity (Kemps and Tiggemann, 2015). Additionally, Veenstra and de Jong (2010) found that restrained eaters have a strong automatic approach tendency for both high-fat and low-fat food while unrestrained eaters do not (Veenstra and de Jong, 2010). It is possible that individuals with obesity and high implicit preferences for high-calorie foods are at higher risk for experiencing unintended time lapses while following dietary prescriptions because they may automatically reach for food even at times when they are not intending to eat a meal or snack.

Lapsing from a diet by eating at an unintended time has been linked with worse weight loss outcomes compared to other types of dietary lapses, i.e., eating a food that was intended to be avoided and eating a larger portion than planned (Goldstein et. al., 2018), so interventions that target triggers for unintended time lapses could be especially effective in improving weight-loss success. Inhibition Control Training is promising for improving weight-loss outcomes for individuals with high implicit preferences for sweets (Forman et al., 2019), so it may be helpful for people with high implicit preferences for high-calorie food as well. If future research replicates our findings showing a relation between IAT and unintended time lapses, Inhibition Control Training may be beneficial for improving weight-loss outcomes by reducing the association between hedonic food and pleasure. This could potentially weaken approach tendencies toward highcalorie food and thus reduce dietary lapses, specifically unintended time lapses.

Surprisingly, there were no associations between baseline IAT for high-calorie foods and eating avoided food or eating larger portion lapses. The results of the study were somewhat inconsistent with findings from other studies. Conner et al. (2007) found that implicit preference for sweets correlated with self-reported sweet consumption and that implicit preference for chocolate correlated with selecting chocolate over fruit. This suggests that implicit preferences for certain foods is associated with consumption and selection of that food item. However, participants were not on a dietary prescription, so they were not instructed to minimize sweets and chocolate. It may be that implicit preferences play a role in food consumption when people are not dieting.

There are several limitations to the study that must be acknowledged. Information provided in the EMA surveys was all self-reported, so responses may be prone to inaccuracies due to misreported details or forgotten lapses. The sample was mostly Caucasian females, so the results from this study might not be generalized to other populations. Although EMA compliance was $83.2 \%$, future studies may benefit from utilizing incentives to achieve high compliance rates to maximize data collection.

With replication, this study can provide insight into how implicit preference for high-calorie food may increase an in- dividual's risk for experiencing specific types of dietary lapse. Future studies can examine the association between implicit preference for high-calorie food and approach tendencies towards palatable food to investigate a potential mechanism underlying the relationship between strong implicit preference for high-calorie food and unintended time dietary lapses. Future research could also explore how skills targeting this implicit subconscious process may prevent unintended time lapses. Because current literature examining the relation between implicit preferences for food and eating habits among participants on dietary prescriptions is limited, future studies can investigate the influence of automatic processes on food choices within the context of behavioral weight loss interventions. It is important to continue researching determinants of dietary lapses to refine tailoring of interventions for weight loss.

\section{ACKNOWLEDGMENTS}

This study was funded by grants from the National Institute for Diabetes and Digestive and Kidney Diseases (ROI DK095069) to Dr. Forman.

\section{CONFLICT OF INTEREST}

Dr. Forman reports royalties from Oxford Press for two workbooks describing acceptance-based behavioral treatment. None of the remaining authors report a conflict of interest.

\section{REFERENCES}

Banse, R., Seise, J., and Zerbes, N. (2001). Implicit attitudes towards homosexuality: Reliability, validity, and controllability of the IAT. Zeitschrift für Experimentelle Psychologie, 48(2), 145-160. Available: 10.1026//0949-3946.48.2.145

Caleyachetty, R., Thomas, G. N., Toulis, K. A., Mohammed, N., Gokhale, K. M., Balachandran, K., and Nirantharakumar, K. (2017). Metabolically healthy obese and incident cardiovascular disease events among 3.5 million men and women. Journal of the American College of Cardiology, 70(12), 1429-1437. Available: 10.1016/j.jacc.2017.07.763.

Carels, R. A., Douglass, O. M., Cacciapaglia, H. M., and O'Brien, W. H. (2004). An ecological momentary assessment of relapse crises in dieting. Journal of Consulting and Clinical Psychology, 72(2), 341. Available: https://doi.org/10.1037/0022-006X.72.2.341.

Carels, R. A., Hoffman, J., Collins, A., Raber, A. C., Cacciapaglia, H., and O'Brien, W. H. (2001). Ecological momentary assessment of temptation and lapse in dieting. Eating Behaviors, 2(4), 307-321. Available: https://doi.org/10.1016/S1471-0153(01)00037-X.

Center for Disease Control and Prevention. (2017). National Health and Nutrition Examination Survey Data. Retrieved August 27, 2019 from https://www.cdc.gov/nchs/fastats/obesity-overweight.htm.

Clerkin, E. M. and Teachman, B. A. (2010). Training implicit social anxiety associations: An experimental intervention. Journal of Anxiety Disorders, 24(3), 300-308. Available: 10.1016/j.janxdis.2010.01.001.

Conner, M. T., Perugini, M., O'Gorman, R., Ayres, K., and Prestwich, A. (2007). Relations between implicit and explicit measures of attitudes and measures of behavior: Evidence of moderation by individual difference variables. Personality and Social Psychology Bulletin, 33(12), 1727-1740. Available: https://doi.org/10.1177/0146167207309194.

Egloff, B., and Schmukle, S. C. (2002). Predictive validity of an implicit association test for assessing anxiety. Journal of Personality and Social Psychology, 83(6), 1441. Available: https://doi.org/10.1037/00223514.83.6.1441. 
Finlayson, G., Bryant, E., Blundell, J. E., and King, N. A. (2009). Acute compensatory eating following exercise is associated with implicit hedonic wanting for food. Physiology and Behavior, 97(1), 62-67. Available: 10.1016/j.physbeh.2009.02.002.

Fishbach, A., and Shah, J. Y. (2006). Self-control in action: implicit dispositions toward goals and away from temptations. Journal of Personality and Social Psychology, 90(5), 820. Available: https://doi. org/10.1037/0022-3514.90.5.820.

Forman, E. M., Butryn, M. L., Manasse, S. M., Crosby, R. D., Goldstein, S. P., Wyckoff, E. P., and Thomas, J. G. (2016). Acceptance based versus standard behavioral treatment for obesity: Results from the mind your health randomized controlled trial. Obesity, 24(10), 2050-2056. Available: 10.1002/oby.21601.

Forman, E. M., Goldstein, S. P., Zhang, F., Evans, B. C., Manasse, S. M., Butryn, M. L., Juarascio, A. S., Abichandani, P., Martin, G. J., and Foster, G. D. (2018). OnTrack: development and feasibility of a smartphone app designed to predict and prevent dietary lapses. Translational Behavioral Medicine, 9(2), 236-245. Available: 10.1093/tbm/iby016.

Forman, E. M., Manasse, S. M., Dallal, D. H., Crochiere, R. J., Loyka, C. M., Butryn, M. L., Juarascio, A. S., and Houben, K. (2019). Computerized neurocognitive training for improving dietary health and facilitating weight loss. Journal of Behavioral Medicine, 1-12. Available: 10.1007/ s10865-019-00024-5.

Forman, E. M., Schumacher, L. M., Crosby, R., Manasse, S. M., Goldstein, S. P., Butryn, M. L., Wyckoff, E. P., and Graham Thomas, J. (2017). Ecological momentary assessment of dietary lapses across behavioral weight loss treatment: characteristics, predictors, and relationships with weight change. Annals of Behavioral Medicine, 51(5), 741-753. Available: 10.1007/s12160-017-9897-x.

Goldstein, S. P., Dochat, C., Schumacher, L. M., Manasse, S. M., Crosby, R. D., Thomas, J. G., Butryn, M. L., and Forman, E. M. (2018). Using ecological momentary assessment to better understand dietary lapse types. Appetite, 129, 198-206. Available: https://doi.org/10.1016/j.appet.2018.07.003

Hoefling, A. and Strack, F. (2008). The tempting effect of forbidden foods. High calorie content evokes conflicting implicit and explicit evaluations in restrained eaters. Appetite, 51(3), 681-689. Available:.

Hofmann, W., Rauch, W., and Gawronski, B. (2007). And deplete us not into temptation: Automatic attitudes, dietary restraint, and self-regulatory resources as determinants of eating behavior. Journal of Experimental Social Psychology, 43(3), 497-504. Available: 10.1016/j. appet.2008.06.004.

Kemps, E. and Tiggemann, M. (2015). Approach bias for food cues in obese individuals. Psychology and Health, 30(3), 370-380. Available: https:// doi.org/10.1080/08870446.2014.974605.

Lowe, M. R. (2003). Self regulation of energy intake in the prevention and treatment of obesity: is it feasible?. Obesity Research, 11(S10), 44S59S. Available: 10.1038/oby.2003.223.

Nederkoorn, C., Houben, K., Hofmann, W., Roefs, A., and Jansen, A. (2010). Control yourself or just eat what you like? Weight gain over a year is predicted by an interactive effect of response inhibition and implicit preference for snack foods. Health Psychology, 29(4), 389. Available: 10.1037/a0019921.

Nosek, B. A., Greenwald, A. G., and Banaji, M. R. (2005). Understanding and using the Implicit Association Test: II. Method variables and construct validity. Personality and Social Psychology Bulletin, 31(2), 166180. Available: $10.1177 / 0146167204271418$.

Perugini, M. (2005). Predictive models of implicit and explicit attitudes. British Journal of Social Psychology, 44(1), 29-45. Available: https://doi. org/10.1348/014466604X23491.

Pulgaron, E. R. (2013). Childhood obesity: a review of increased risk for physical and psychological comorbidities. Clinical Therapeutics, 35(1), A18-A32. Available: 10.1016/j.clinthera.2012.12.014.

Shiv, B. and Fedorikhin, A. (1999). Heart and mind in conflict: The interplay of affect and cognition in consumer decision making. Journal of Consumer Research, 26(3), 278-292. Available: https://doi. org/10.1086/209563.
Shiv, B. and Fedorikhin, A. (2002). Spontaneous versus controlled influences of stimulus-based affect on choice behavior. Organizational Behavior and Human Decision Processes, 87(2), 342-370. Available: https://doi.org/10.1006/obhd.2001.2977.

Veenstra, E. M. and de Jong, P. J. (2010). Restrained eaters show enhanced automatic approach tendencies towards food. Appetite, 55(1), 30-36. Available: 10.1016/j.appet.2010.03.007.

Williams, E. P., Mesidor, M., Winters, K., Dubbert, P. M., and Wyatt, S. B. (2015). Overweight and obesity: prevalence, consequences, and causes of a growing public health problem. Current Obesity Reports, 4(3), 363-370. Available: 10.1007/s13679-015-0169-4. 